\title{
Vibrissa-Signaled Eyeblink Conditioning Induces Somatosensory Cortical Plasticity
}

\author{
Roberto Galvez, Craig Weiss, Aldis P. Weible, and John F. Disterhoft \\ Department of Physiology and Institute for Neuroscience, Northwestern University Feinberg School of Medicine, Chicago, Illinois 60611
}

Whisker deflection conditioned stimuli (CS) were demonstrated to activate physiologically and anatomically defined barrels in the contralateral somatosensory cortex and to support trace- eyeblink conditioned responses when paired with corneal airpuff unconditioned stimuli in rabbits. Analysis of cytochrome-oxidase-stained somatosensory whisker-associated cortical barrels revealed a rowspecific expansion of the conditioned compared with the nontrained hemisphere. This expansion was not evident in pseudo-conditioned rabbits, suggesting that this expansion of conditioned cortical barrels in response to a hippocampal- and forebrain-dependent learning task (trace conditioning) is associative rather than activity dependent. Using whisker stimulation as a CS in the well studied eyeblink conditioning paradigm will facilitate characterizing sensory cortical involvement in controlling and modulating an associatively learned response at the neural systems and cellular level.

Key words: whisker; barrel; PMBSF; cytochrome oxidase; trace conditioning; hippocampus

\section{Introduction}

Eyeblink conditioning is one of the oldest and best-studied associative learning paradigms (Gormezano et al., 1962). During training, a neutral conditioned stimulus (CS) is paired with a salient unconditioned stimulus (US), eliciting an unconditioned response (UR). After repeated CS-US pairings, the subject begins to exhibit a learned conditioned response (CR) that precedes the US. In trace conditioning, the CS and US are temporally separated by a stimulus-free interval. This form of conditioning is dependent on the hippocampus (Solomon et al., 1986; Moyer et al., 1990; Kim et al., 1995; Weiss et al., 1999; Takehara et al., 2002); however, once learned, the hippocampus is no longer necessary to perform the task (Kim et al., 1995; Takehara et al., 2002), suggesting that long-term consolidation occurs elsewhere. However, little is known concerning the specific site(s) for long-term memory storage of trace associations.

One of the more likely candidate locations for long-term memory storage is the cerebral cortex (Squire et al., 2004). Various studies have suggested the caudal anterior cingulate (cAC) as one possible cortical site (Kronforst-Collins and Disterhoft, 1998). However, a recent analysis suggested that cAC is involved in attending to the CS (Weible et al., 2003), rather than serving as a storage site. Another cortical region that could play a role in long-term memory storage of trace associations would be the primary sensory cortical region that perceives the CS (Kraus and Disterhoft, 1981, 1982; Weinberger et al., 1984, 1993)

Sensory cortical regions have been demonstrated to exhibit

Received 0ct. 15, 2005; revised April 27, 2006; accepted April 28, 2006.

This work was supported by Mechanisms of Aging and Dementia Training Grants T32 AG20506 and R01 MH47340.

Correspondence should be addressed to Roberto Galvez, Department of Physiology, Northwestern University Feinberg School of Medicine, 303 East Chicago Avenue, Chicago, IL 60611. E-mail: r-galvez@northwestern.edu. DOI:10.1523/JNEUROSCI.5582-05.2006

Copyright $\odot 2006$ Society for Neuroscience $\quad$ 0270-6474/06/266062-07\$15.00/0 experience-dependent plasticity using various paradigms. For example, focal retinal lesions induce a topographic reorganization in primary visual cortex (Darian-Smith and Gilbert, 1994, 1995). Likewise, training on an object orientation task alters both sensitivity and preferred orientation in primary visual cortex (Schoups et al., 2001; Ghose et al., 2002). Furthermore, frequency discrimination training alters the preferred frequency cortical receptive field in primary auditory cortex (Disterhoft and Stuart, 1976; Kitzes et al., 1978; Diamond and Weinberger, 1986, 1989; Edeline et al., 1993; Recanzone et al., 1993; Rutkowski and Weinberger, 2005). Finally, tactile discrimination alters somatosensory cortical map hand representation (Jenkins et al., 1990; Recanzone et al., 1992) and neuronal firing in primary somatosensory barrel cortex (Krupa et al., 2004) for digit and whisker stimulation, respectively. These and similar studies suggest that the primary sensory cortex is modulated in response to learning and could be one site among others that mediate long-term memory storage.

Our trace-conditioning paradigm used the well characterized vibrissa system. In the vibrissa system, tactile information from individual whisker deflections is sent to cortex in a one-to-one orientation via a tri-synaptic pathway (facial nerve to medullary barrelets/thalamic barreloids/somatosensory barrel cortex) (Woolsey and Van der Loos, 1970). The following analyses demonstrated that cytochrome oxidase (CO) staining can be used to visualize electrophysiologically confirmed, whisker-associated cortical barrels in adult rabbits. We then showed that whisker stimulation is a suitable CS for trace conditioning, as it is for delay conditioning (Das et al., 2001). Finally, we demonstrated trace conditioning induced alterations in barrel cortical map representations.

\section{Materials and Methods}

Cortical barrel localization

Surgery. Three-month-old female New Zealand White rabbits weighing $3.0-4.0 \mathrm{~kg}$ were used. The surgical procedures adhered to the guidelines 
of Northwestern University's Animal Care and Use committee. Ketamine $(60 \mathrm{mg} / \mathrm{kg})$ and xylazine $(10 \mathrm{mg} / \mathrm{kg})$ were administered intramuscularly to anesthetize the rabbits for surgery. Eyes were kept moist with a thin layer of antibacterial ophthalmic ointment.

For single-neuron recordings, the rabbit was positioned in a stereotaxic frame, with lambda $1.5 \mathrm{~mm}$ below bregma. The skull was exposed and two $5.0-\mathrm{mm}$-diameter openings centered $2.5 \mathrm{~mm}$ posterior and 7.5 $\mathrm{mm}$ lateral from bregma were made, exposing the intact dura. Heart rate and temperature were monitored, and supplemental anesthesia administered as needed.

Single-neuron recording. Neuronal data were recorded using the Cheetah system (Neuralynx, Tucson, AZ). Tetrodes consisted of four Formvar-coated nichrome wires ( $25 \mu \mathrm{m}$ diameter bare; $37 \mu \mathrm{m}$ diameter coated), which were bonded at one end with epoxylite and exhibited an average impedance of $0.5-1.5 \mathrm{M} \Omega$. Neuronal activity was passed to a headstage amplifier (HS-27) via a customized adapter, buffered with the HS-27, filtered $(600-6000 \mathrm{~Hz})$, and amplified $(20,000 \times)$.

Penetration into the barrel fields was verified via manual vibrissa stimulation and auditory monitoring of neuronal activity. During neuronal recording, vibrissa stimulation was performed as described for behavioral training (see below, Whisker-trace conditioning), with stimulation applied every 4-6 s. During stimulation of B-row whiskers, neuronal activity was recorded at multiple locations within the contralateral barrel cortex. After each recording period, the activity of individual neurons was isolated using Neuralynx Spike Sort software, and peri-event time histograms (PETHs) were generated off-line to identify stimulus-specific changes in neuronal firing rate. $z$ scores were then calculated for the PETHs to identify significant changes $(z>1.96)$ in firing rate compared with a $1000 \mathrm{~ms}$ prestimulus baseline. When neurons exhibiting robust stimulus-specific increases in firing rate were identified, a current (DC, $+10 \mu \mathrm{A}, 1 \mathrm{~s}$ duration) was passed through a single channel of the tetrode to mark the site for subsequent histological identification. This procedure was performed in both hemispheres.

Rabbits were then killed by intravenous administration of a lethal dose of sodium pentobarbital $(97.5 \mathrm{mg} / \mathrm{kg})$ and perfused transcardially with PBS, pH 7.4, followed by $2 \%$ paraformaldehyde for a light fixation. The cerebral cortex was then dissected off, flattened (Strominger and Woolsey, 1987), and postfixed overnight in $4 \%$ paraformaldehyde. Flattened cortices were then cryoprotected in 30\% sucrose in PBS and sectioned $(30 \mu \mathrm{m})$ parallel to the surface with a cryostat.

Cytochrome oxidase/Nissl staining. For electrode placement after single-neuron recording, alternating sections were stained for cytochrome oxidase for barrel visualization or Nissl for electrode placement. For CO staining, free-floating sections were placed in staining solution $(0.05 \% \mathrm{DAB}, 0.03 \%$ cytochrome $c, 4 \%$ sucrose in $\mathrm{PBS})$ at $37^{\circ} \mathrm{C}$ for $6 \mathrm{~h}$ to ensure consistent staining. CO staining was terminated via a series of PBS washes, and sections were then mounted onto gelatin-coated slides for analysis. For Nissl staining, sections were mounted onto slides and stained according to a standard cresyl violet staining protocol.

\section{Whisker-trace conditioning}

Surgery. The surgical preparation was performed as outlined above (Cortical barrel localization, Surgery). Thirteen rabbits were surgically implanted with an atraumatic restraining device for head immobilization during training as described previously (Disterhoft et al., 1977).

Conditioning. Training was conducted while rabbits were restrained in a cloth bag in a Plexiglas box with their heads immobilized. After $1 \mathrm{~d}$ of habituation, seven rabbits began trace conditioning and seven rabbits began pseudo-conditioning. Trace conditioning consisted of 80 daily trials with a pseudo-randomly varied intertrial interval (ITI) of 30-60 s. Each trial consisted of a $250 \mathrm{~ms}$ CS followed by a stimulus-free $500 \mathrm{~ms}$ trace interval immediately followed by a $150 \mathrm{~ms}$ US. For the CS, whiskers B1-B6 were attached $1.0-1.5 \mathrm{~cm}$ from the rabbit's face to an applicator made of card stock paper that was then attached to a flat piezoceramic strip (T220-A4-303; Piezo Systems, Cambridge, MA). Great care was taken to ensure that the piezoceramic strip did not come into contact with any of the nonconditioned whiskers. To generate the CS, $120 \mathrm{~V} \mathrm{AC}$ was delivered to the piezoceramic strip generating a $60 \mathrm{~Hz}, 130 \mu \mathrm{m}$ dorsal-ventral deflection of the attached whiskers (Das et al., 2001).
Straddler whiskers have not been characterized in rabbits; thus, for whisker labeling, we adopted the nomenclature used for rabbits by Gould (1986). The US was a 3.5 psi airpuff to the eye. A CR, closure of the nictitating membrane monitored via change in voltage from an infrared reflective sensor, was defined as a $4 \mathrm{SD}$ change in voltage above baseline present within $20 \mathrm{~ms}$ of US onset. Rabbits were killed $24 \mathrm{~h}$ after reaching behavioral criterion, defined as exhibiting CRs on $80 \%$ of the trials. Pseudo-conditioned rabbits were presented with 80 CS and 80 US random unpaired trials with a pseudo-randomly varied 15-30 s ITI. Pseudoconditioned rabbits never reached criterion; thus, to determine when they should be terminated from the experiment, they were yoked to a trace-conditioned rabbit and killed $24 \mathrm{~h}$ after the trace-conditioned rabbit reached criterion. Yoked rabbits were trained, killed, tissue processed, and analyzed together. One pseudo-conditioned rabbit was eliminated from the analysis because of difficulty finding cortical barrels on both hemispheres. Rabbits were killed and their cortices were processed as described above (Cortical barrel localization, Single-neuron recording).

Cytochrome oxidase. For barrel analysis after trace conditioning, every third section was stained for cytochrome oxidase. This staining protocol usually resulted in three sections containing cortical barrels. In such situations, the middle section was used for analysis. If barrels were only visible on two sections, the section that had the better defined barrels was used. Cortical barrels were typically observed starting at $683.33 \pm 124.4$ $\mu \mathrm{m}$ from the surface of the cortex and spanned ventrally $326 \pm 113.85$ $\mu \mathrm{m}$. CO staining was conducted as outlined above (Cortical barrel localization, Cytochrome oxidase/Nissl staining).

Barrel cortical analysis. To determine barrel length and width, cytochrome oxidase stained cortical barrels were scanned at constant illumination on a flat bed scanner at 5000 dots per inch (dpi). For barrel length analysis (the total length or anteroposterior extent of barrels 1-3 within a row), a $63-\mu \mathrm{m}$-wide sampling rectangle was placed over a single row of barrels, ensuring that the ends of the rectangle extended beyond the first and last barrel using NIH ImageJ, version 1.32J (http://rsb.info.nih.gov/ ij/). An optical density histogram of the area within the rectangle was then generated, again using ImageJ to determine the location for barrel walls. The length of the first to the third barrel was recorded (see Fig. $4 \mathrm{~A}$ ). For barrel width analysis (the mediolateral distance of the barrel, perpendicular to the row), a $63-\mu \mathrm{m}$-wide sampling rectangle was placed over a single barrel, ensuring that the ends of the rectangle extended into the barrel in the adjacent row using ImageJ (http://rsb.info.nih.gov/ij/). Using ImageJ, an optical density histogram of the area within the rectangle was generated, and the width of the barrel (wall to wall) was measured for the first three barrels in rows A-D. To ensure consistent sampling as a result of animal, whisker row, and sectioning variability in the number of stained barrels per row, only the first three barrels in rows A, B, C, and D were measured. Although this method illustrated the point between two cortical barrel walls (see Fig. $4 A$, tic marks on $x$-axis), it was not sensitive enough to delineate the barrel hollows and walls separately.

To assist in determining whether it was the hollows or walls that were changing, barrel hollow and wall boundaries for the first three barrels in row $\mathrm{B}$ on cytochrome-oxidase-stained sections were visualized on an Olympus (Melville, NY) microscope attached to an IBM-compatible computer, traced, and the areas determined via Stereo Investigator (MicroBrightField, Colchester, VT). For optical density analysis, cytochrome-oxidase-stained slides were scanned at constant illumination at 5000 dpi. Optical density values were acquired from these electronic images by randomly sampling three equally sized areas from the barrel hollow and wall via NIH ImageJ, version 1.32J (http://rsb.info. nih.gov/ij/). All analyses were conducted blind to the training condition and cortical hemisphere.

Statistics. For behavioral analysis, the percentage conditioned response was analyzed using a mixed general linear model (GLM)-ANOVA on Statistical Analysis Software (SAS) (SPSS, Chicago, IL) with days of training as within-subject and training group as between-subject variables. For length, width, area, and optical density analyses, neuroanatomical mean values were tested using a mixed-model GLM-ANOVA on SAS with row and hemisphere as within-subject and training group as between-subject variables. 


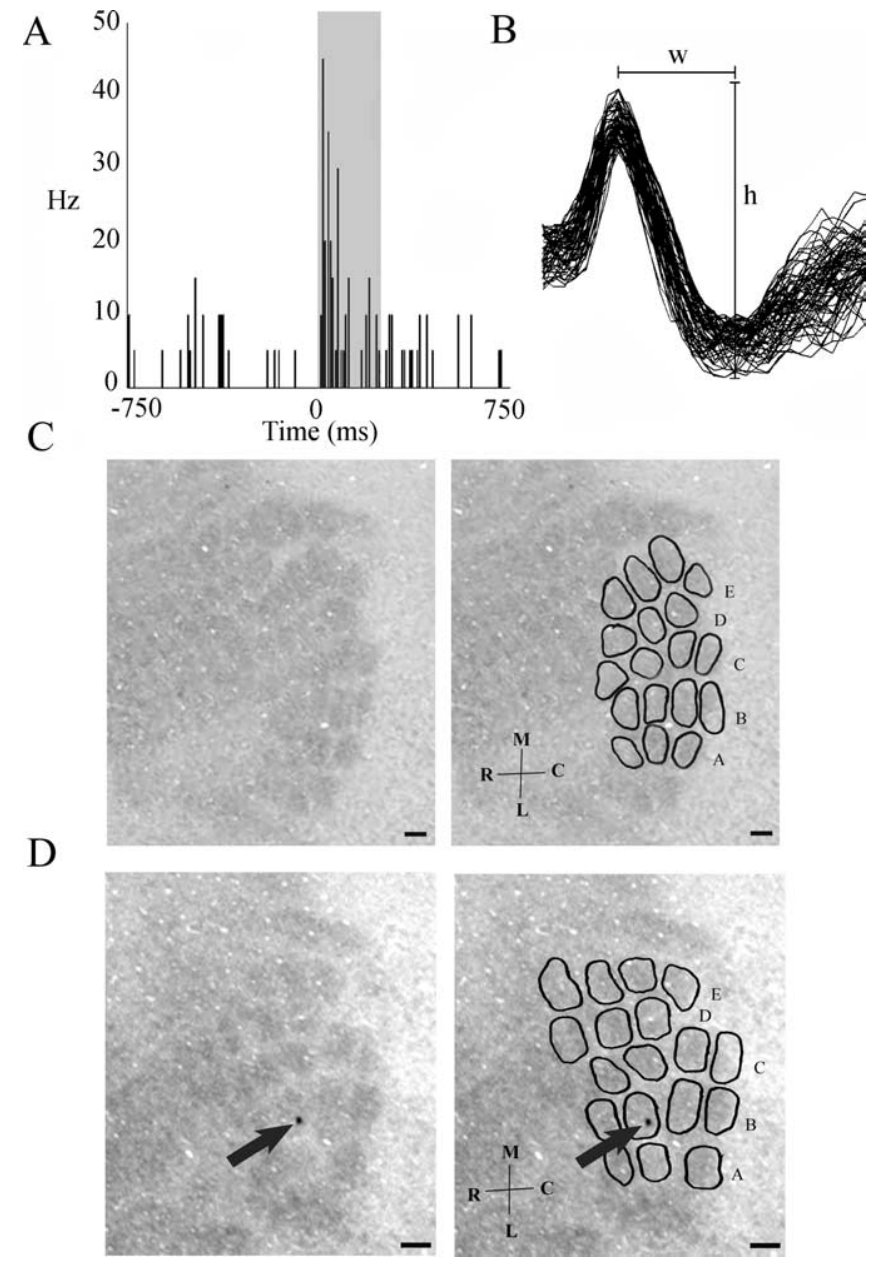

Figure 1. Cytochrome-oxidase-stained cortical barrel representations correspond to whiskers on the rabbit's face. $\boldsymbol{A}$, Extracellular cortical single unit activity in response to $250 \mathrm{~ms}$ stimulation of B-row whiskers (gray box corresponds to the time of stimulation). Electrode coordinates from dura and cortical surface were $8.0 \mathrm{~mm}$ lateral, $2.75 \mathrm{~mm}$ posterior, and $1.3 \mathrm{~mm}$ ventral. One bin equals $10 \mathrm{~ms}$. Note the increase in firing rate during whisker stimulation. $\boldsymbol{B}$, Waveforms for the unit represented in $\boldsymbol{A}$ [width (w), $0.33 \mathrm{~ms}$; height (h), $145 \mu \mathrm{V}$ ]. C, Left, Cytochrome-oxidase-stained rabbit barrel cortex. Note the barrel-like pattern. Right, Same image with barrels and rows delineated. $\boldsymbol{D}$, Left, Unaltered cytochrome-oxidase-stained rabbit barrel cortex from which the neuron in $\boldsymbol{A}$ was recorded. Note the marking lesion in barrel $B 3$ (arrow). Right, Same image with barrels and rows delineated. Scale bars, $250 \mu \mathrm{m}$. M, Medial; L, lateral; R, rostral; C, caudal.

\section{Results}

\section{Identification of cortical barrels}

After staining for $\mathrm{CO}$, a barrel-like pattern with darkly stained barrel hollows and lightly stained barrel walls consistent with published rabbit barrel cortical electrophysiological maps (Gould, 1986) was visualized in a subset of tangential sections most likely spanning layer IV of the somatosensory cortex. Single neurons were identified in the contralateral primary somatosensory cortex exhibiting an increase in firing rate after B-row whisker stimulation $(250 \mathrm{~ms})$ (Fig. 1A). The marking lesion for this site, verified via alternating Nissl-stained sections, was located in the second row, third column of a CO barrel-like pattern (Fig. $1 D)$. Stimulation of B-row whiskers failed to elicit a significant increase in firing rate when the electrode was moved $>300 \mu \mathrm{m}$ in the mediolateral plane from the histologically verified recording site (data not shown). These analyses demonstrate that a barrellike pattern can be visualized in adult rabbit somatosensory cortex via $\mathrm{CO}$ staining, and in vivo neuronal activity suggests that this

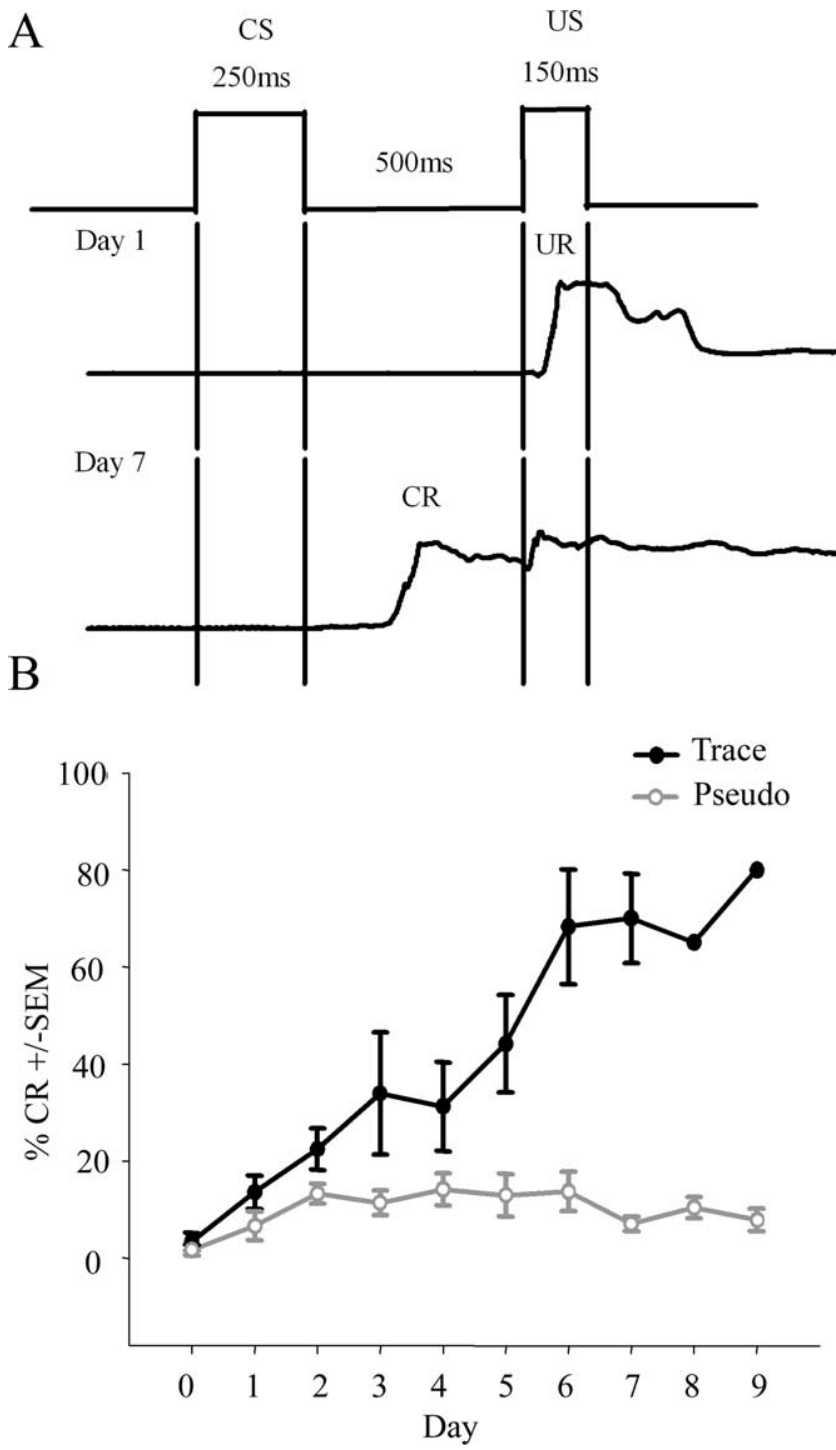

Figure 2. Rabbits can learn trace-eyeblink conditioning with vibrissa stimulation as the conditioned stimulus. A, Typical eyeblink response during conditioning on day 1 and day 7 . The upward deflection represents a sweep of the nictitating membrane across the cornea. Note the $C R$ during the trace interval on day 7 as an indication of learning. $\boldsymbol{B}$, The percentage of number of conditioned responses across training days for trace- and pseudo-conditioned rabbits. Only one conditioned rabbit required $9 \mathrm{~d}$ to reach criterion ( $80 \%$ CRs); hence, there are no error bars for these last two data points. Error bars represent SEM.

pattern corresponds to the whisker pad on the rabbit's face, as described previously in electrophysiological maps of rabbit barrel cortex (Gould, 1986).

\section{Trace conditioning}

Whisker-trace-conditioned rabbits, compared with pseudoconditioned, exhibited a significant increase in CRs (Fig. 2) $\left(F_{(9,80)}=4.53 ; p<0.05\right)$, requiring a mean of $6.3 \pm 0.07 \mathrm{~d}$ to reach criterion. Note that this number of days to reach criterion represents $125 \pm 14 \mathrm{~s}$ of total vibrissa CS stimulation. Post hoc analyses of $\mathrm{CR}$ parameters demonstrated adaptive properties such as a decrease in CR onset $\left(F_{(1,42)}=15.42 ; p<0.05\right)$ and increases in CR duration $\left(F_{(1,42)}=13.94 ; p<0.05\right)$ and area $\left(F_{(1,42)}=14.31 ; p<0.05\right)$ (Fig. 3), as described previously for delay conditioning with whisker stimulation (Das et al., 2001) and for trace conditioning using an auditory CS. A few rabbits 
A
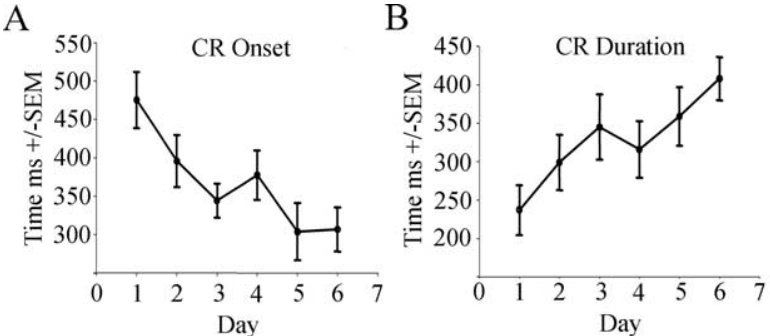

$\mathrm{C}$

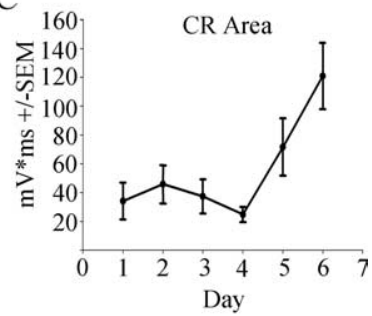

Figure 3. Conditioned response exhibits adaptive properties. $\boldsymbol{A}$, The time of the conditioned response onset across days of training (US onset is at $750 \mathrm{~ms}$ ). $\boldsymbol{B}$, The duration of the conditioned response across days of training. $\boldsymbol{C}$, The area of the conditioned response across days of training. Error bars represent SEM. Time 0 equals $C S$ onset.

A
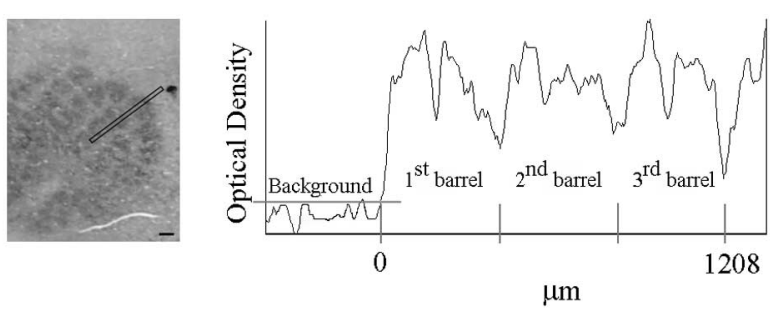

B

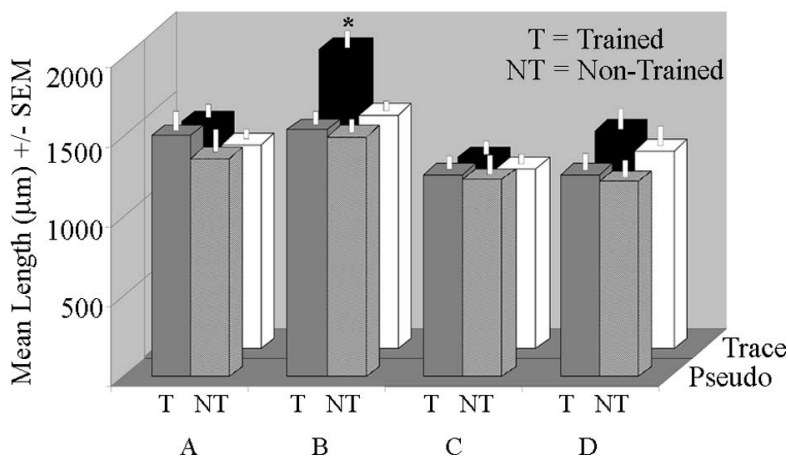

Figure 4. Whisker-eyeblink conditioning increases metabolic representation for conditioned cortical barrels. $A$, Left, Illustration of a cytochrome-oxidase-stained cortical barrel field with a sampling area over the B-row. Scale bar, $250 \mu \mathrm{m}$. Right, Illustration of an optical density histogram of the row of barrels outlined in the barrel field illustration to the left. Note the tic marks on the $x$-axis delineating the point between two barrel walls. $\boldsymbol{B}$, The mean length for the first three cortical barrels in each row on the trained and nontrained hemisphere for trace-and pseudo-conditioned rabbits. Note the increase in length for the trace-conditioned B-row trained hemisphere. Error bars represent SEM. ${ }^{*} p<0.05$.

also exhibited a deflection of the nictitating membrane, which returned to baseline during the first $150 \mathrm{~ms}$ after CS onset, consistent with previously described $\alpha$ responses (Solomon et al., 1986) (data not shown). These $\alpha$ responses only attained a maximum of $9.25 \pm 2.02 \%$ of all trials. Note that, as stated in Materials and Methods (Whisker-trace conditioning, Conditioning), a CR was defined as a $4 \mathrm{SD}$ change in voltage above baseline within 20 ms of US onset; thus, these $\alpha$ responses did not meet this criterion and were not counted as CRs.

\section{Learning-induced plasticity}

Analysis of barrel length (the total length or anteroposterior extent of barrels 1-3 within a row) demonstrated an increase in the mean length for CO-stained trained barrels (B-row) compared with the nontrained hemisphere (within-subject; $F_{(1,11)}=6.61$; $p<0.05)$ or pseudo-controls (between-subject; $F_{(1,12)}=9.94$; $p<0.05$ ) (Fig. 4B). The unconditioned whiskers (rows A, C, and D) of the trained hemisphere did not demonstrate a similar increase in length when compared with the nontrained hemisphere or pseudo-controls, suggesting that this increase (B-row) is specific to learning the conditioned response. No significant differences in barrel length within the same hemisphere by condition were observed. Analysis of barrel width (the mediolateral distance of the barrel, perpendicular to the row) demonstrated no significant change in response to conditioning in conditioned or unconditioned barrels (rows A, $\mathrm{B}, \mathrm{C}$, and $\mathrm{D}$ ). To determine whether the conditioning induced increase in barrel length was caused by changes in the barrel hollow or wall, area analyses of both regions were then conducted. Analysis of B-row hollow size revealed a conditioning induced increase in the mean area compared with the nontrained hemisphere (within-subject; $F_{(1,11)}=$ 12.97; $p<0.05)$ or pseudo-controls (between-subject; $F_{(1,12)}=$ 6.28 ; $p<0.05$ ) (Fig. 5). Analysis of wall area revealed no significant differences (Fig. 5), suggesting that the barrel wall is not altered with conditioning. However, the observed increase in total barrel metabolic area would only require a slight decrease in the thickness of each individual wall surrounding the barrel hollow; thus, we may not have the statistical power to resolve such a slight change in individual wall thicknesses. Analysis of optical density values demonstrated that the barrel hollow was significantly darker than the wall in all subjects (within-subject; $\left.F_{(1,167)}=10.57 ; p<0.05\right)$, but neither the hollow nor wall showed a significant change in optical density in response to conditioning (Table 1). These observations suggest that whisker conditioning does not increase the metabolic activity within a barrel but rather increases the size of the cortical whisker metabolic representation.

\section{Discussion}

Trace-eyeblink conditioning has long been believed to require higher cortical processing. We set out to establish a model system for examining sensory cortical involvement in long-term memory storage of trace associations. In the process, we developed a methodology for visualizing adult rabbit whisker cortical barrels, confirmed via electrophysiological analysis. We also demonstrated that vibrissa stimulation is an effective CS for trace-eyeblink conditioning and that this form of conditioning induces a learning-dependent, row-specific expansion of cortical barrels measured via cytochrome oxidase staining, indicating that it could be a site for long-term memory storage. Note that we are not suggesting that the barrel cortex is the only site for long-term memory storage of whisker-trace conditioning. For example, cerebellar interpositus lesions abolish the ability to acquire or exhibit already acquired trace CRs (Woodruff-Pak et al., 1985; Gerwig et al., 2005), suggesting that it could also play a role in long-term memory storage. However, these analyses demonstrate that not only can the vibrissa system provide a model for further analysis of sensory cortical plasticity in trace-eyeblink conditioning, but because of the topographical map and highly structured neuronal organization of the barrel cortex (Swadlow and Gusev, 2000, 2002), we believe this model system will greatly facilitate our understanding of conditioning-induced cortical plasticity.

Cytochrome oxidase activity in layer IV of the barrel cortex is attributable to metabolic activity in dendritic processes (WongRiley and Welt, 1980) associated with sites of synaptic contact (Kageyama and Wong-Riley, 1982; Mjaatvedt and Wong-Riley, 
1988). Cortical barrel hollows in layer IV receive input from corresponding whisker related barreloids in the ventral posterior medial (VPM) thalamus. Thus, the conditioning induced increase in CO-stained cortical barrel hollows could be caused by reorganization or activation of either VPM or intracortical projections. Regardless of the source of the plasticity (VPM or intracortical), both would require either axonal, dendritic, or synaptic proliferation and/or functional activation of pre-existing processes to elicit the conditioning induced row specific CO cortical barrel map expansion.

In support of a mechanism requiring synaptogenesis or functional activation of pre-existing processes, studies have demonstrated that whisker stimulation induces synaptic plasticity. For example, whisker stimulation has been shown to increase the total number of synapses (Knott et al., 2002) and specifically increase the number of inhibitory synapses for at least $4 \mathrm{~d}$ after stimulation (Knott et al., 2002) in the barrel cortex. Furthermore, partial vibrissectomy in adult mice increases cortical 2-deoxyglucose (2DG) barrel representation (Siucinska and Kossut, 1994; Kossut and Juliano, 1999) and axonal density in layer IV of the barrel cortex for the spared whiskers (Kossut and Juliano, 1999), suggesting an increase in synapses. Likewise, whisker chessboard deprivation, a paradigm in which the whiskers are removed in a chessboard pattern, induces synaptic plasticity in layer V barrel cortical neurons (Trachtenberg et al., 2002). These studies suggest that the observed conditioning induced expansion of the $\mathrm{CO}$ cortical barrel map representation is caused by either synaptogenesis and/or functional activation of pre-existing synapses.

In further support for a synaptic explanation for the observed plasticity, various studies have demonstrated an absence of experience-induced remodeling of dendritic fields in the adult barrel cortex. For example, adult spiny stellate neurons along the barrel wall in layer IV of the barrel cortex exhibit barrel hollow orientated dendritic fields (Woolsey et al., 1975; Simons and Woolsey, 1984; Greenough and Chang, 1988), which can be altered with whisker follicle damage before (Harris and Woolsey, 1979, 1981; Steffen and Van der Loos, 1980) but not after postnatal day 4 in mice (Harris and Woolsey, 1979, 1981). Furthermore, whisker chessboard deprivation, shown to induce synaptic plasticity, does not significantly alter dendritic morphology on layer V barrel neurons (Trachtenberg et al., 2002). These studies suggest that adult whisker deprivation is unlikely to induce large scale remodeling of the dendritic fields and further support a synaptic theory for adult barrel cortical plasticity.

We should note that although dendritic growth appears to be an unlikely explanation for our observations, we do not feel it can be completely ruled out. Increased sensory stimulation via environmental enrichment increases occipital cortical dendritic material (Volkmar and Greenough, 1972; Greenough et al., 1979; Withers and Greenough, 1989). Furthermore, many proteins such as the fragile $\mathrm{X}$ mental retardation protein (a cargo protein that regulates mRNA translation at the synapse) (Miyashiro et al., 2003) have been shown to play a role in both spine maturation (Nimchinsky et al., 2001; Galvez and Greenough, 2005) and pruning of dendritic processes (Galvez et al., 2003, 2005), sug-

Barrel hollow

race-conditione

seudo-conditioned

Nontrained

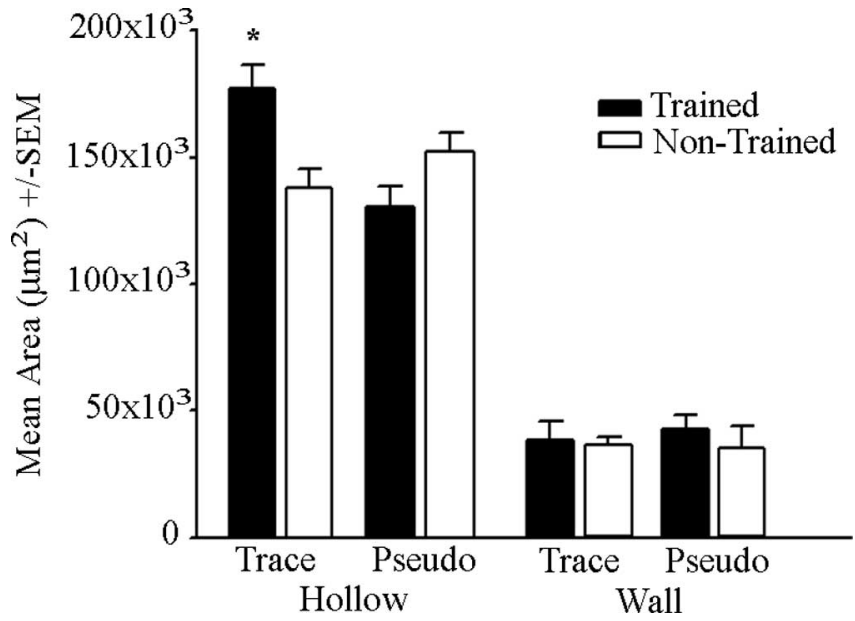

Figure 5. Whisker-eyeblink conditioning increases metabolic representation for conditioned cortical barrel hollows. The mean area for the barrel hollow and wall for B-row on the trained and nontrained hemisphere for trace- and pseudo-conditioned rabbits is shown. Note the increase in hollow area for the trace-conditioned B-row trained hemisphere. Error bars represent SEM. ${ }^{*} p<0.05$.

Table 1. Mean optical density values \pm SEM for trace- and pseudo-conditioned rabbits on the trained and nontrained hemisphere for the barrel hollow and wall of rows $A, B, C$, and D

A $B$ C C D

Trained

$56.09 \pm 3.34$

$56.16 \pm 3.20$

$55.67 \pm 3.49$

$56.58 \pm 6.49$

$56.68 \pm 6.23$

$54.02 \pm 3.12$

$56.45 \pm 6.01$

$61.62 \pm 10.97$

$59.68 \pm 10.41$

$55.68 \pm 6.04$

$65.06 \pm 14.34$

$53.16 \pm 6.66$

$52.84 \pm 6.48$

$55.43 \pm 6.63$

$51.76 \pm 6.79$

$50.16 \pm 2.70$

$51.58 \pm 5.55$

$49.75 \pm 2.64$

$51.16 \pm 5.66$

$46.76 \pm 2.68$

$49.76 \pm 5.18$

$52.58 \pm 9.12$

$51.97 \pm 8.28$

$49.68 \pm 5.40$

$55.95 \pm 12.75$

$47.40 \pm 6.20$

$47.04 \pm 5.95$

$49.00 \pm 6.00$

$45.59 \pm 5.57$

$46.84 \pm 6.73$

ker cortical barrel rows stained with cytochrome oxidase after trace conditioning or pseudo-conditioning.

gesting that the same or similar mechanisms regulate synaptic and dendritic growth. Thus, dendritic growth is a plausible hypothesis that also warrants further investigation.

Angiogenesis could also expand CO stained cortical barrels. However, unlike dendritic proliferation, we do not believe angiogenesis could account for the observed plasticity. Angiogenesis, although shown to have positive effects on cognitive performance (Churchill et al., 2002), is typically associated with physical activity and not learning (Black et al., 1990). Thus, if the expansion was caused by activity-induced angiogenesis, one would expect to observe expansion in the barrels of pseudo-conditioned animals as well.

Finally, gliogenesis could also be a source for the observed cortical barrel expansion. However, like angiogenesis, we do not believe this could account for the observed plasticity. Gliogenesis, although shown to be increased with enriched rearing (Altman and Das, 1964; Diamond et al., 1964, 1966; Szeligo and Leblond, 1977; Bhide and Bedi, 1984a,b; Sirevaag and Greenough, 1991), requires at least $30 \mathrm{~d}$ to induce a significant change (Sirevaag and Greenough, 1991). Our observed increase occurred on average after only $6 \mathrm{~d}$ of training. This time period is well below that shown to be required for inducing gliogenesis. 
The present study is not the first to demonstrate that the adult whisker barrel cortex remains plastic in adulthood. For example, whisker pairing has been shown to alter adult receptive fields for the remaining whiskers (Diamond et al., 1993; Armstrong-James et al., 1994). Furthermore, vibrissa stimulation paired with footshock or a water reward in mice increases the size of the 2DG cortical barrel representation for the conditioned barrels (Siucinska and Kossut, 1996, 2004). Finally, studies have demonstrated that the adult CO barrel cortical map representation can also be modulated (Machin et al., 2004; Polley et al., 2004). These studies found that when adult rats were exposed to an enriched environment, the receptive field size in layer II/III decreased (Polley et al., 2004), whereas the size of the overall CO-stained cortical barrel field in layer IV increased (Machin et al., 2004; Polley et al., 2004). These studies, along with our findings, clearly demonstrate that not only does the barrel cortex retain the ability to be modulated in adulthood, but that under certain conditions, the adult CO cortical barrel map representation can also be modulated.

We should note that unlike these previous analyses, which used long periods of whisker stimulation, our paradigm induced barrel cortical plasticity over a considerably shorter time span (125 $\pm 14 \mathrm{~s}$; sum total of vibrissa CSs for the average number of trials to reach criterion in our study). Furthermore, our analysis is the first to demonstrate that CO staining can visualize adult rabbit whisker-related cortical barrels and that barrel cortical plasticity is associated with vibrissa CS processing during a well characterized, hippocampal- and forebrain-dependent associative learning task, trace-eyeblink conditioning.

Sensory cortical involvement in trace conditioning, to our knowledge, has not been extensively investigated. The current study established a methodology for visualizing adult rabbit whisker-related cortical barrels. Although parvalbumin staining of adult rabbit somatosensory cortex has been shown to reveal a barrel-like pattern (McMullen et al., 1994), this pattern has not been confirmed electrophysiologically to correspond to whiskers on the rabbit's face. Note that cortical barrels cannot be visualized using standard Nissl stains in adult rabbits (Rice et al., 1985). Our analysis electrophysiologically demonstrated that the observed cytochrome oxidase barrel pattern corresponds to the whiskers on the rabbit's face. Furthermore, our analyses demonstrated that vibrissa stimulation is a suitable CS for trace-eyeblink conditioning and that this form of conditioning induces sensory cortical plasticity. With this model system for examining sensory cortical changes associated with trace conditioning now established, future analyses will explore the mechanism(s) for this learning specific plasticity.

\section{References}

Altman J, Das GD (1964) Autoradiographic examination of the effects of enriched environment on the rate of glial multiplication in the adult rat brain. Nature 204:1161-1163.

Armstrong-James M, Diamond ME, Ebner FF (1994) An innocuous bias in whisker use in adult rats modifies receptive fields of barrel cortex neurons. J Neurosci 14:6978-6991.

Bhide PG, Bedi KS (1984a) The effects of a lengthy period of environmental diversity on well-fed and previously undernourished rats. I. Neurons and glial cells. J Comp Neurol 227:296-304.

Bhide PG, Bedi KS (1984b) The effects of a lengthy period of environmental diversity on well-fed and previously undernourished rats. II. Synapse-toneuron ratios. J Comp Neurol 227:305-310.

Black JE, Isaacs KR, Anderson BJ, Alcantara AA, Greenough WT (1990) Learning causes synaptogenesis, whereas motor activity causes angiogenesis, in cerebellar cortex of adult rats. Proc Natl Acad Sci USA $87: 5568-5572$.

Churchill JD, Galvez R, Colcombea S, Swain RA, Kramer AF, Greenough WT
(2002) Exercise, experience and the aging brain. Neurobiol Aging 5691:1-15.

Darian-Smith C, Gilbert CD (1994) Axonal sprouting accompanies functional reorganization in adult cat striate cortex. Nature 368:737-740.

Darian-Smith C, Gilbert CD (1995) Topographic reorganization in the striate cortex of the adult cat and monkey is cortically mediated. J Neurosci 15:1631-1647.

Das S, Weiss C, Disterhoft JF (2001) Eyeblink conditioning in the rabbit (Oryctolagus cuniculus) with stimulation of the mystacial vibrissae as a conditioned stimulus. Behav Neurosci 115:731-736.

Diamond DM, Weinberger NM (1986) Classical conditioning rapidly induces specific changes in frequency receptive fields of single neurons in secondary and ventral ectosylvian auditory cortical fields. Brain Res 372:357-360.

Diamond DM, Weinberger NM (1989) Role of context in the expression of learning-induced plasticity of single neurons in auditory cortex. Behav Neurosci 103:471-494.

Diamond MC, Krech D, Rosenzweig MR (1964) The effects of an enriched environment on the histology of the rat cerebral cortex. J Comp Neurol 123:111-120.

Diamond MC, Law F, Rhodes H, Lindner B, Rosenzweig MR, Krech D, Bennett EL (1966) Increases in cortical depth and glia numbers in rats subjected to enriched environment. J Comp Neurol 128:117-126.

Diamond ME, Armstrong-James M, Ebner FF (1993) Experiencedependent plasticity in adult rat barrel cortex. Proc Natl Acad Sci USA 90:2082-2086.

Disterhoft JF, Stuart DK (1976) Trial sequence of changed unit activity in auditory system of alert rat during conditioned response acquisition and extinction. J Neurophysiol 39:266-281.

Disterhoft JF, Kwan HH, Lo WD (1977) Nictitating membrane conditioning to tone in the immobilized albino rabbit. Brain Res 137:127-143.

Edeline JM, Pham P, Weinberger NM (1993) Rapid development of learning-induced receptive field plasticity in the auditory cortex. Behav Neurosci 107:539-551.

Galvez R, Greenough WT (2005) Sequence of abnormal dendritic spine development in primary somatosensory cortex of a mouse model of the fragile X mental retardation syndrome. Am J Med Genet A 135:155-160.

Galvez R, Gopal AR, Greenough WT (2003) Somatosensory cortical barrel dendritic abnormalities in a mouse model of the fragile $\mathrm{X}$ mental retardation syndrome. Brain Res 971:83-89.

Galvez R, Smith RL, Greenough WT (2005) Olfactory bulb mitral cell dendritic pruning abnormalities in a mouse model of the Fragile-X mental retardation syndrome: further support for FMRP's involvement in dendritic development. Dev Brain Res 157:214-216.

Gerwig M, Haerter K, Hajjar K, Dimitrova A, Maschke M, Kolb FP, Thilmann AF, Gizewski ER, Timmann D (2005) Trace eyeblink conditioning in human subjects with cerebellar lesions. Exp Brain Res:1-15.

Ghose GM, Yang T, Maunsell JH (2002) Physiological correlates of perceptual learning in monkey V1 and V2. J Neurophysiol 87:1867-1888.

Gormezano I, Schneiderman N, Deaux E, Fuentes I (1962) Nictitating membrane: classical conditioning and extinction in the albino rabbit. Science 138:33-34.

Gould III HJ (1986) Body surface maps in the somatosensory cortex of rabbit. J Comp Neurol 243:207-233.

Greenough WT, Chang FL (1988) Dendritic pattern formation involves both oriented regression and oriented growth in the barrels of mouse somatosensory cortex. Brain Res 471:148-152.

Greenough WT, Juraska JM, Volkmar FR (1979) Maze training effects on dendritic branching in occipital cortex of adult rats. Behav Neural Biol 26:287-297.

Harris RM, Woolsey TA (1979) Morphology of golgi-impregnated neurons in mouse cortical barrels following vibrissae damage at different postnatal ages. Brain Res 161:143-149.

Harris RM, Woolsey TA (1981) Dendritic plasticity in mouse barrel cortex following postnatal vibrissa follicle damage. J Comp Neurol 196:357-376.

Jenkins WM, Merzenich MM, Ochs MT, Allard T, Guic-Robles E (1990) Functional reorganization of primary somatosensory cortex in adult owl monkeys after behaviorally controlled tactile stimulation. J Neurophysiol 63:82-104.

Kageyama GH, Wong-Riley MT (1982) Histochemical localization of cytochrome oxidase in the hippocampus: correlation with specific neuronal types and afferent pathways. Neuroscience 7:2337-2361. 
Kim JJ, Clark RE, Thompson RF (1995) Hippocampectomy impairs the memory of recently, but not remotely, acquired trace eyeblink conditioned responses. Behav Neurosci 109:195-203.

Kitzes LM, Farley GR, Starr A (1978) Modulation of auditory cortex unit activity during the performance of a conditioned response. Exp Neurol 62:678-697.

Knott GW, Quairiaux C, Genoud C, Welker E (2002) Formation of dendritic spines with GABAergic synapses induced by whisker stimulation in adult mice. Neuron 34:265-273.

Kossut M, Juliano SL (1999) Anatomical correlates of representational map reorganization induced by partial vibrissectomy in the barrel cortex of adult mice. Neuroscience 92:807-817.

Kraus N, Disterhoft JF (1981) Location of rabbit auditory cortex and description of single unit activity. Brain Res 214:275-286.

Kraus N, Disterhoft JF (1982) Response plasticity of single neurons in rabbit auditory association cortex during tone-signalled learning. Brain Res 246:205-215.

Kronforst-Collins MA, Disterhoft JF (1998) Lesions of the caudal area of rabbit medial prefrontal cortex impair trace eyeblink conditioning. Neurobiol Learn Mem 69:147-162.

Krupa DJ, Wiest MC, Shuler MG, Laubach M, Nicolelis MA (2004) Layerspecific somatosensory cortical activation during active tactile discrimination. Science 304:1989-1992.

Machin R, Blasco B, Bjugn R, Avendano C (2004) The size of the whisker barrel field in adult rats: minimal nondirectional asymmetry and limited modifiability by chronic changes of the sensory input. Brain Res 1025:130-138.

McMullen NT, Smelser CB, Rice FL (1994) Parvalbumin expression reveals a vibrissa-related pattern in rabbit SI cortex. Brain Res 660:225-231.

Miyashiro KY, Beckel-Mitchener A, Purk TP, Becker KG, Barret T, Liu L, Carbonetto S, Weiler IJ, Greenough WT, Eberwine J (2003) RNA cargoes associating with FMRP reveal deficits in cellular functioning in Fmr 1 null mice. Neuron 37:417-431.

Mjaatvedt AE, Wong-Riley MT (1988) Relationship between synaptogenesis and cytochrome oxidase activity in Purkinje cells of the developing rat cerebellum. J Comp Neurol 277:155-182.

Moyer Jr JR, Deyo RA, Disterhoft JF (1990) Hippocampectomy disrupts trace eye-blink conditioning in rabbits. Behav Neurosci 104:243-252.

Nimchinsky EA, Oberlander AM, Svoboda K (2001) Abnormal development of dendritic spines in FMR1 knock-out mice. J Neurosci 21:5139-5146.

Polley DB, Kvasnak E, Frostig RD (2004) Naturalistic experience transforms sensory maps in the adult cortex of caged animals. Nature 429:67-71.

Recanzone GH, Merzenich MM, Jenkins WM, Grajski KA, Dinse HR (1992) Topographic reorganization of the hand representation in cortical area $3 \mathrm{~b}$ owl monkeys trained in a frequency-discrimination task. J Neurophysiol 67:1031-1056.

Recanzone GH, Schreiner CE, Merzenich MM (1993) Plasticity in the frequency representation of primary auditory cortex following discrimination training in adult owl monkeys. J Neurosci 13:87-103.

Rice FL, Gomez C, Barstow C, Burnet A, Sands P (1985) A comparative analysis of the development of the primary somatosensory cortex: interspecies similarities during barrel and laminar development. J Comp Neurol 236:477-495.

Rutkowski RG, Weinberger NM (2005) Encoding of learned importance of sound by magnitude of representational area in primary auditory cortex. Proc Natl Acad Sci USA 102:13664-13669.

Schoups A, Vogels R, Qian N, Orban G (2001) Practising orientation identification improves orientation coding in V1 neurons. Nature 412:549-553.

Simons DJ, Woolsey TA (1984) Morphology of Golgi-Cox-impregnated barrel neurons in rat SmI cortex. J Comp Neurol 230:119-132.

Sirevaag AM, Greenough WT (1991) Plasticity of GFAP-immunoreactive astrocyte size and number in visual cortex of rats reared in complex environments. Brain Res 540:273-278.
Siucinska E, Kossut M (1994) Short term changes of cortical body maps following partial vibrissectomy in adult mice. Acta Neurobiol Exp (Wars) 54:345-354.

Siucinska E, Kossut M (1996) Short-lasting classical conditioning induces reversible changes of representational maps of vibrissae in mouse SI cortex-a 2DG study. Cereb Cortex 6:506-513.

Siucinska E, Kossut M (2004) Experience-dependent changes in cortical whisker representation in the adult mouse: a 2-deoxyglucose study. Neuroscience 127:961-971.

Solomon PR, Vander Schaaf ER, Thompson RF, Weisz DJ (1986) Hippocampus and trace conditioning of the rabbit's classically conditioned nictitating membrane response. Behav Neurosci 100:729-744.

Squire LR, Stark CE, Clark RE (2004) The medial temporal lobe. Annu Rev Neurosci 27:279-306.

Steffen H, Van der Loos H (1980) Early lesions of mouse vibrissal follicles: their influence on dendrite orientation in the cortical barrelfield. Exp Brain Res 40:419-431.

Strominger RN, Woolsey TA (1987) Templates for locating the whisker area in fresh flattened mouse and rat cortex. J Neurosci Methods 22:113-118

Swadlow HA, Gusev AG (2000) The influence of single VB thalamocortical impulses on barrel columns of rabbit somatosensory cortex. J Neurophysiol 83:2802-2813.

Swadlow HA, Gusev AG (2002) Receptive-field construction in cortical inhibitory interneurons. Nat Neurosci 5:403-404.

Szeligo F, Leblond CP (1977) Response of the three main types of glial cells of cortex and corpus callosum in rats handled during suckling or exposed to enriched, control and impoverished environments following weaning. J Comp Neurol 172:247-263.

Takehara K, Kawahara S, Takatsuki K, Kirino Y (2002) Time-limited role of the hippocampus in the memory for trace eyeblink conditioning in mice. Brain Res 951:183-190.

Trachtenberg JT, Chen BE, Knott GW, Feng G, Sanes JR, Welker E, Svoboda $\mathrm{K}$ (2002) Long-term in vivo imaging of experience-dependent synaptic plasticity in adult cortex. Nature 420:751-752.

Volkmar FR, Greenough WT (1972) Rearing complexity affects branching of dendrites in the visual cortex of the rat. Science 176:1145-1147.

Weible AP, Weiss C, Disterhoft JF (2003) Activity profiles of single neurons in caudal anterior cingulate cortex during trace eyeblink conditioning in the rabbit. J Neurophysiol 90:599-612.

Weinberger NM, Hopkins W, Diamond DM (1984) Physiological plasticity of single neurons in auditory cortex of the cat during acquisition of the pupillary conditioned response: I. Primary field (AI). Behav Neurosci 98:171-188.

Weinberger NM, Javid R, Lepan B (1993) Long-term retention of learninginduced receptive-field plasticity in the auditory cortex. Proc Natl Acad Sci USA 90:2394-2398.

Weiss C, Bouwmeester H, Power JM, Disterhoft JF (1999) Hippocampal lesions prevent trace eyeblink conditioning in the freely moving rat. Behav Brain Res 99:123-132.

Withers GS, Greenough WT (1989) Reach training selectively alters dendritic branching in subpopulations of layer II-III pyramids in rat motorsomatosensory forelimb cortex. Neuropsychologia 27:61-69.

Wong-Riley MT, Welt C (1980) Histochemical changes in cytochrome oxidase of cortical barrels after vibrissal removal in neonatal and adult mice. Proc Natl Acad Sci USA 77:2333-2337.

Woodruff-Pak DS, Lavond DG, Thompson RF (1985) Trace conditioning: abolished by cerebellar nuclear lesions but not lateral cerebellar cortex aspirations. Brain Res 348:249-260.

Woolsey TA, Van der Loos H (1970) The structural organization of layer IV in the somatosensory region (SI) of mouse cerebral cortex. The description of a cortical field composed of discrete cytoarchitectonic units. Brain Res 17:205-242.

Woolsey TA, Dierker ML, Wann DF (1975) Mouse SmI cortex: qualitative and quantitative classification of golgi-impregnated barrel neurons. Proc Natl Acad Sci USA 72:2165-2169. 